\title{
POLARIZATION AND N-N ELASTIC SCATTERING AMPLITUDES *
}

\author{
Gordon P. Ramsey \\ Physics Department, Loyola University Chicago, Chicago, IL 60626 \\ and
}

High Energy Physics Division, Argonne National Laboratory, Argonne, IL 60439

\begin{abstract}
We discuss the role of polarization measurements and scattering amplitudes for elastic nucleon-nucleon processes at high energy. The relative normalization of these amplitudes involves a "leading order form factor" which we determine empirically. These amplitudes provide an economical description of a large body of existing data and make some nontrivial predictions for spin observables. In particular, we have investigated cross sections and asymmetry data at large angles (including $90^{\circ}$ c.m.) and the fixed $|t|$, large $s$ region, dominated by the three-gluon exchange mechanism. Our results indicate that polarization experiments can test basic QCD elements such as helicity conservation, wave function properties and the interplay between various interaction mechanisms. The significance of this analysis will be discussed in terms of specific polarization experiments, which can be performed at Fermilab and Brookhaven (RHIC. AGS).
\end{abstract}

- Paper presented at the 11th International Symposium on High Energy Spin Physics, Indiana University, September, 1994. Work supported by the U.S. Department of Energy, Division of High Energy Physics, Contract W-31-109-ENG-38. 


\section{DISCLAIMER}

Portions of this document may be illegible in electronic image products. Images are produced from the best available original document. 


\section{Phenomenological Normalization of the Spin Amplitudes}

The study of exclusive hard scattering processes provides an interesting theoretical challenge. Existing data on fixed-angle differential cross sections and spin observables at high energies suggest that predictions made using the constituent counting rules are accurate in describing exclusive hard scattering and that the data may be in a kinematic regime where hadronic amplitudes can be calculated perturbatively. There exist difficulties in the calculations (1) for $p p \rightarrow p p$ scattering which imply that there is little chance of a reliable "first principles" calculation based on QCD perturbation theory. However, experimentally, high intensity proton beams and a clean signature for the elastic scattering process make this one of the best studied set of experimental cross sections. Spin dependent observables and the spin-averaged cross section are accurately measured over a wide range of kinematic variables (2). Thus, for a given helicity amplitude, (3) it is possible to do a phenomenological analysis where the normalization of the amplitude is fit to data at one point while its $s$ and $t$ dependence are extracted from the theoretical calculation. This approach leads to a large number of asymptotic predictions, but there has been considerable discussion about which of the involved mechanisms are important.

We define the observables for $N N \rightarrow N N$ elastic scattering in terms of the JacobWick helicity amplitudes: $\Phi_{1}(s, t)$ through $\Phi_{5}(s, t)(3)$. Other helicity amplitudes are related to these independent ones using parity conservation, time-reversal invariance and identical particle symmetry.

The differential cross section in terms of these amplitudes is: (4)

$$
\frac{d \sigma}{d t}=\frac{\pi}{2 s\left(s-4 m^{2}\right)}\left[\left|\Phi_{1}\right|^{2}+\left|\Phi_{2}\right|^{2}+\left|\Phi_{3}\right|^{2}+\left|\Phi_{4}\right|^{2}+4\left|\Phi_{5}\right|^{2}\right]
$$

where the total cross section is given by: $\Sigma=s\left(s-4 m^{2}\right) \frac{d \sigma}{d t}$.

We assume that we can separate a soft, coherent Regge contribution for each independent amplitude, which dominates the observables at small $t$. We also assume there exists a "hard" component for each amplitude which obeys the Brodsky-Lepage factorization at large $t$. The coherent Regge components should be exponentially suppressed at large $t$, reflecting the size of the individual proton. The overall amplitudes at large $t$ then consist primarily of the Landshoff and Quark-Interchange components (4). This separation is largely a matter 
of convention. The $s$ and $t$ dependence of the Landshoff and QIM amplitudes are specified by simple QCD calculations, and the assumption is that the proton is well described by its minimal three-quark Fock state. A consistent normalization of the theoretical amplitudes demands that the scattering is dominated by a "quark interchange" mechanism in kinematic regions where sharp structure has been observed.

Donnachie and Landhoff (5) have done a thorough phenomenological study of the differential cross sections for $p p$ and $p \bar{p}$ at high energies. The normalization of the Landshoff 3 -gluon mechanism amplitude can be reliably specified at high energy and fixed- $t$ from elastic scattering data at the ISR collider. From the known kinematic dependence of the Landshoff amplitude, we can extrapolate its effect to the energies of the ANL data. The differential cross section at ISR energies can be pametrized as:

$$
\frac{d \sigma}{d t}=\pi \frac{|L(t)|^{2}}{\left(2 m_{p}^{2}-t\right)^{8}}\left[1+8|\epsilon|^{2} \frac{(-t)}{\left(2 m_{p}^{2}-t\right)^{2}}+\cdots\right]
$$

where the second term in brackets is associated with $\left|\Phi_{5}\right|^{2}$ and other terms are suppressed by powers of $\frac{t}{s}$. Comparing equation 1.2 with data at large $s$ and with $-t$ outside the coherent Regge region is the first step in a phenomenological normalization. At large $-t$, $\left(\geq 4 \mathrm{GeV}^{2}\right)$, the three gluon exchange mechanism dominates. In this kinematic regime, there is strong experimental support for an approximately energy-independent component of the cross section which behaves as $t^{-8}$. Experimentally, the numerical value extracted for $|L(t)|^{2}$ from these data can be used to normalize this mechanism.

Note that the $|t|$ range of these high energy collider data overlaps with the $|t|-$ range of the low energy large angle data where structure has been observed in the twospin observable $A_{N N}$ and the $90^{\circ} \mathrm{c} . \mathrm{m}$. differential cross section (6). Since the Landshoff normalization factors $|L(t)|$ are dependent only upon $-t$, we can use existing cross section data at various $s$ for comparable $-t$ values to phenomenologically determine their behavior. We can then extrapolate the Landshoff amplitudes to the energies of the $90^{\circ} \mathrm{c} . \mathrm{m}$. data to find their effect on the behavior of the cross section and $A_{N N}$. We have fit elastic $p p$ data from the Argonne ZGS and CERN (ISR) for various $\sqrt{ } s$ ranging from $3 \mathrm{GeV} / \mathrm{c}$ to $62 \mathrm{GeV} / \mathrm{c}$ and for a common range of $-t$.

Our average fit to the the ISR data, for the differential cross section, which is relatively 
independent of $s$, is

$$
\frac{d \sigma}{d t}\left(\mu b / G e V^{2}\right)=1.6 \exp [-1.7|t|] .
$$

The ANL data (7) at $90^{\circ} \mathrm{c} . \mathrm{m}$. covers a range of $\sqrt{ } s$ from 3.3 to $5.1 \mathrm{GeV} / \mathrm{c}$. There is a marked change in structure of the cross section for $-t$ near $7(\mathrm{GeV} / \mathrm{c})^{2}$. Our fit to the data is:

$$
\begin{aligned}
\frac{d \sigma}{d t}\left(\mu b / G e V^{2}\right) & =6836 \exp [-1.59|t|], & & 3.8 \leq-t \leq 6.8(\mathrm{GeV} / \mathrm{c})^{2} \\
& =24 \exp [-0.76|t|], & & 7.3 \leq-t \leq 11.3(\mathrm{GeV} / \mathrm{c})^{2}
\end{aligned}
$$

If we now assume the ISR data are in a region dominated by the Landshoff mechanism, we can then calculate a ratio of the cross sections between the ISR and ANL data in the mid- $t$ region:

$$
\left(\frac{d \sigma}{d t}\right)_{I S R} /\left(\frac{d \sigma}{d t}\right)_{A N L} \approx\left(2.4 \times 10^{-4}\right) \exp [-0.12|t|] .
$$

When considering the effects of the Landshoff mechanism at $90^{\circ} \mathrm{cm}$, the $u$-channel terms become important, but are but are correctly accounted for in our parametrization. Thus at ANL energies, the total Landshoff contribution is at most $3 \times 10^{-4}$ for this range of $t$ values. Thus, although the Landshoff contribution to the ISR cross section may be large, its effect on the ANL $90^{\circ}$ data is down by a factor of $10^{-4}$. We conclude that another mechanism must be responsible for the structure of the data in this region. Given the known $s$ dependence of the three-gluon amplitude, we can easily rule out the possibility that the Landshoff mechanism is involved in these low energy structures.

\section{Structure of the Amplitudes at $90^{\circ}$ c.m.}

There are two distinct types of phenomena which have been observed in the $90^{\circ} \mathrm{c} . \mathrm{m}$. cross section for $p p$ elastic scattering: (1) oscillations in $s^{10 \frac{d \sigma}{d t}}$ and (2) structure in $A_{N N}$, both as a function of energy.

It is possible to use the symmetries which occur at $90^{\circ}$ in the $\mathrm{cm}$ to do a simple amplitude analysis which can extract the basic features of the sub-asymptotic mechanism responsible for the structure observed. This may have important ramifications for studies of nuclear transparency and for the study of other exclusive processes. 
Since the Landshoff normalization, as determined from ISR data, rules out the possibility that this mechanism is responsible for this "low energy" behavior, it appears that we must search elsewhere for the explanation of these unusual structures. We consider that the basic symmetries of the QIM model are reflected in the large angle amplitudes and that the structures may be corrections to these amplitudes. Due to the symmetries at $90^{\circ} \mathrm{c} . \mathrm{m}$.. we have the kinematic constraints that $\Phi_{5}=0$ and $\Phi_{4}=-\Phi_{3}$. Also, the double helicity-flip amplitude, $\Phi_{2}$, can be neglected at the energies where the data exist. Helicity conservation therefore implies that the observables at $90^{\circ}$ can be understood in terms of only two independent amplitudes. For the observables we consider here, the expressions are

$$
\begin{aligned}
\Sigma & =\frac{1}{2}\left[\left|\Phi_{1}\right|^{2}+2\left|\Phi_{3}\right|^{2}\right] \\
A_{N N} & =\frac{2\left|\Phi_{3}\right|^{2}}{\left[\left|\Phi_{1}\right|^{2}+2\left|\Phi_{3}\right|^{2}\right]} .
\end{aligned}
$$

We now implement the constraint that the amplitudes approach the QIM result: $\Phi_{1}^{Q}=2 \Phi_{3}^{Q}$ at $90^{\circ}$ c.m., to write

$$
\begin{aligned}
& \Phi_{1}=2 \Phi^{Q}+\hat{\Phi}_{1} \\
& \Phi_{3}=\Phi^{Q}+\hat{\Phi}_{3},
\end{aligned}
$$

where $\Phi^{Q}$ is a smooth power-law behaved amplitude which characterizes the asymptotic observables, while $\hat{\Phi}_{1}$ and $\hat{\Phi}_{3}$ are sub-asymptotic corrections. If we write

$$
\Sigma_{0} \equiv \frac{1}{2} 6\left|\Phi^{Q}\right|^{2}
$$

then

$$
\begin{gathered}
A_{N N}\left(\Sigma / \Sigma_{0}\right)=\frac{1}{3} \frac{\left|\Phi_{3}\right|^{2}}{\left|\Phi^{Q}\right|^{2}}=\frac{1}{3} \frac{\left|\Phi^{Q}+\hat{\Phi}_{3}\right|^{2}}{\left|\Phi^{Q}\right|^{2}} \equiv \frac{1}{3} R_{3} \\
\left(1-A_{N N}\right) \Sigma / \Sigma_{0}=\frac{1}{6} \frac{\left|\Phi_{1}\right|^{2}}{\left|\Phi^{Q}\right|^{2}}=\frac{2}{3} \frac{\left|\Phi^{Q}+\frac{1}{2} \hat{\Phi}_{1}\right|^{2}}{\left|\Phi^{Q}\right|^{2}} \equiv \frac{2}{3} R_{1}
\end{gathered}
$$

where $R_{1} \equiv \frac{\left|\Phi_{1}\right|^{2}}{\left|\Phi_{1}^{Q}\right|^{2}}$ and $R_{3} \equiv \frac{\left|\Phi_{3}\right|^{2}}{\left|\Phi_{3}^{Q}\right|^{2}}$. The data show interference effects in both amplitudes with the structure in $\Phi_{1}$ occurring at a lower energy than that in $\Phi_{3}$. A plot of these amplitudes is shown in figure 1 . 
The structure of $A_{N N}$ for the range $4<|t|<7 \mathrm{GeV}^{2}$ is relatively flat. The value of $A_{N N}$ however, differs significantly from the QIM prediction of $\frac{1}{3}$. Given the average value of $A_{N N}$ from the data, equation 2.4 implies that $R_{1} \approx 9 R_{3}$. If the QIM mechanism were dominant, we would observe that $R_{1} \approx 4 R_{3}$. We can conclude that the additional sub-asymptotic mechanism appears to enhance the process described by $\Phi_{1}$ over that of $\Phi_{3}$ significantly at these energies. The differential cross section in this region exhibits a relatively steady $(-t)^{-10}$ behavior. If we make the Ansatz that $\Sigma_{0}=3.5 \times 10^{8} /(-t)^{10}$, then $\Sigma$ has the same $t$ behavior and $\left|\Phi_{1}\right|^{2} /\left|\Phi_{1}^{Q}\right|^{2} \approx 3$. This is a measure of the relative strength of the mechanism which "interferes" with the QIM amplitudes here to cause $A_{N N}$ to dip below the QIM prediction.

In the region $|t|>7 \mathrm{GeV}^{2}$, the slope of the cross section changes and $A_{N N}$ simultaneously begins to rise sharply. The differential cross section exhibits more of a $t^{-8}$ behavior in this region. The amplitude $\Phi_{3}$ also becomes more significant here, since $\frac{\left|\Phi_{1}\right|^{2}}{\left|\Phi_{3}\right|^{2}} \approx 1$ for $|t| \geq 8 \mathrm{GeV}^{2}$. The mechanism responsible for the change in slope of the cross section and the rapid rise in $A_{N N}$ will then couple more strongly to $\Phi_{3}$ than in the other region. This may not be the same mechanism responsible for the behavior of the data in the lower $-t$ region. Eventually, the mechanism interfering with $\Phi_{3}$ dominates the QIM contributions in a way to cause $A_{i N N}$ to rise well above the QIM prediction.

We have not discussed the specific dynamical mechanisms which may be responsible for these effects, except to rule out a significant contribution from the Landshoff threegluon exchange diagrams discussed above. Brodsky and deTeramond, (8) have discussed the structure of $A_{N N}$ in terms of a specific di-baryon resonance, which couples to $\Phi_{3}$, but not to $\Phi_{1}$. The structure of these amplitudes indicates that existing data are not in a regime where "nuclear transparency" should be a feature of $p A \rightarrow p p(A-1)$. Thus it is important to push for experiments at higher energy on nuclear targets to see if nuclear transparency emerges in this region. The work of Jain and Ralston (9) implies that the subasymptotic corrections are suppressed in the nuclear environment. The point-like cross section, $\Sigma_{0}$ is a more useful quantity for understanding nuclear effects in their approach than is the physical cross section. An important requirement for understanding these effects is to seek new data at higher energies, such as at RHIC. 


\section{The Polarization Asymmetry at Large Energies}

The structure of the elastic amplitudes within the framework of the constituent based hard-scattering model can be severely constrained by measurements of the elastic polarization asymmetry at large $s$ and for $|t| \geq 4 \mathrm{GeV}^{2}$. In the traditional Regge theory approach, the polarization asymmetry vanishes at large $s$. In contrast, the hard-scattering approach allows for possible helicity-flip effects associated with the hadronic wave function. These effects vanish at large $|-t|$, but the helicity-flip amplitudes can share the same $s$ dependence as the helicity conserving ones. This leads to a polarization asymmetry which is almost $s$-independent and hence, falls off only as a function of $t$.

We can write the polarization asymmetry in terms of the helicity amplitudes. Our parameterization of the helicity-flip amplitude, $\Phi_{5}$, is given as a combination of the helicityconserving amplitudes and is based on the idea of restructuring a proton wave function from scattered quarks which are approximately collinear. This approach builds in the constraint of hadronic helicity conservation so that at fixed angles, the polarization asymmetry vanishes. The $-t$ dependence is chosen to match dimensional counting rules. In this approach, the polarization asymmetry becomes independent of energy at fixed $t$. We can reproduce the existing polarization data very well. Details of this work will appear in a forthcoming publication. The behavior of the polarization at large energies can be summarized as:

(1) small $|t|$ : There is an overall $(-t)^{\frac{1}{2}}$ factor and the polarization should fall off with energy, reflecting the coherent behavior of factorizable Regge poles.

(2) large $|t|$ : Outside the coherent region, the polarization becomes asymptotically energy-independent and should behave like $(-t)^{-\frac{1}{2}}$ at large $s$.

Those polarization measurements which exist (10) are consistent with these predicted regularities. Further measurements of the polarization asymmetry at various energies, which could be done at Fermilab and at RHIC, are needed to test these underlying principles.

\section{Summary and Conclusions}

We have used the normalization of the Landshoff model helicity amplitudes which 
are taken from the phenomenological studies mentioned above and continued the amplitudes to smaller $s$ values. There is little uncertainty in this exercise since the $s$ dependence of the Landshoff amplitudes are well specified by the model. The continuation shows that the Landshoff amplitudes are too small to be involved in the oscillations observed in $d \sigma / d t$ at $90^{\circ} \mathrm{c} . \mathrm{m}$. or in the sharp structure observed in $A_{N N}$. The most natural explanation of these striking phenomena involves the interference of some asymptotic mechanism with a dominant QIM model amplitude set.

Using the symmetries in the amplitudes at $90^{\circ}$ c.m., we have expressed the data in terms of $\Phi_{1}$ and $\Phi_{3}$. The data suggest a structure which interferes with the dominant QIM amplitudes. This interference occurs at a lower energy in $\Phi_{1}$ than in $\Phi_{3}$, but is approximately the same magnitude in each amplitude. The data disappear in a kinematic regime where there is a lot of structure and it would be interesting to continue these measurements at some higher energies. There exists a real opportunity to do these measurements at the Brookhaven AGS with the addition of a partial Siberian snake to allow for polarized beams. It will be interesting to see whether the data approach the value of $A_{N N}=\frac{1}{3}$ as predicted by the QIM model and whether the cross section oscillations fade away at higher energy.

The other measurement which can provide new insight involves the single-spin polarization asymmetry. Our simple model relates different amplitudes using a spin-flip "form factor" involving a small parameter related to SU(6) breaking. Experiments which could be performed at Fermilab and Brookhaven would further test the validity of this model in the hard scattering region: $m_{p}^{2} \ll-t \ll s$.

Finally, within the context of our model, we have looked at the data for evidence of non-trivial behavior of the observables associated with the running of the QCD coupling or with the falloff of Sudhakov form factor. (11) We could find no evidence of these effects. This may indicate that while constituent based models can provide important insight into the structure of $p p \rightarrow p p$ amplitudes, the data are not in a kinematic regime where full perturbative calculations can be attempted.

\section{Acknowledgements}

This paper is based on work done with D. Sivers. We are grateful for advice and suggestions from A. White, S. Brodsky, J. Ralston and C. Carlson. 


\section{References}

1. G.R. Farrar, H. Zhang, A.A. Globlin and I.R. Zhitnitsky, Nucl. Phys. B311, 585 (1989); G.R. Farrar, E. Maina, and F. Neri, Phys. Rev. Lett. 53, 28 (1984) and ibid., 742(1984).

2. A. Bohm, et. al., Phys. Lett. 49B, 491 (1974); E. Nagy, et. al., Nucl. Phys. B150, 221 (1979). Foley, et.al., Phys. Rev. Lett. 15, 45 (1965); C.W. Akerlof, et. al., Phys. Rev. 159, 1138 (1967); R.C. Kammerud, et. al., Phys. Rev. D4, 1309 (1971).

3. M. Jacob and G.C. Wick, Ann. Phys.7, 404 (1959).

4. G. P. Ramsey and D. Sivers, Phys. Rev. D45, 79 (1992) and Phys. Rev. D47, 93 (1993); G.R. Farrar, et. al. Phys. Rev. D20, 202 (1979); S.J. Brodsky, et.al., Phys. Rev. D20, 2278 (1979).

5. A. Donnachie and P.V. Landshoff., Z. Phys. C 2, 55 (1979); Phys. Lett. 123B, 345 (1983); Nucl. Phys. B231, 189 (1983); B244, 322 (1984); B267, 690 (1986).

6. A. Bohm, et. al., Phys. Lett. 49B, 491 (1974); E. Nagy, et. al., Nucl. Phys. B150, 221 (1979).

7. C.W. Akerlof, et. al., Phys. Rev. 159, 1138 (1967); R.C. Kammerud, et. al., Phys. Rev. D4, 1309 (1971).

8. S.J. Brodsky and G. deTeramond, Phys. Rev. Lett. 60, 1924 (1988).

9. P. Jain and J.P. Ralston, KUHEP 93-47. Proc. of Int. Conf. on elastic and diffractive scattering, 5th Blois Conf., Providence RI, Jun 93.

10. G.W. Abshire, et. al., Phys. Rev. Lett. 32, 1261 (1974).

11. V. Sudhakov, Zh. Eksp. Theor. Phis. 30, 87 (1956) and Sov. Phys. JETP 3, 65 (196.5); J. Botts and G. Sterman, Phys. Lett. B224, 201 (19S9) and Nucl. Phys. B325, $62(1989)$. 


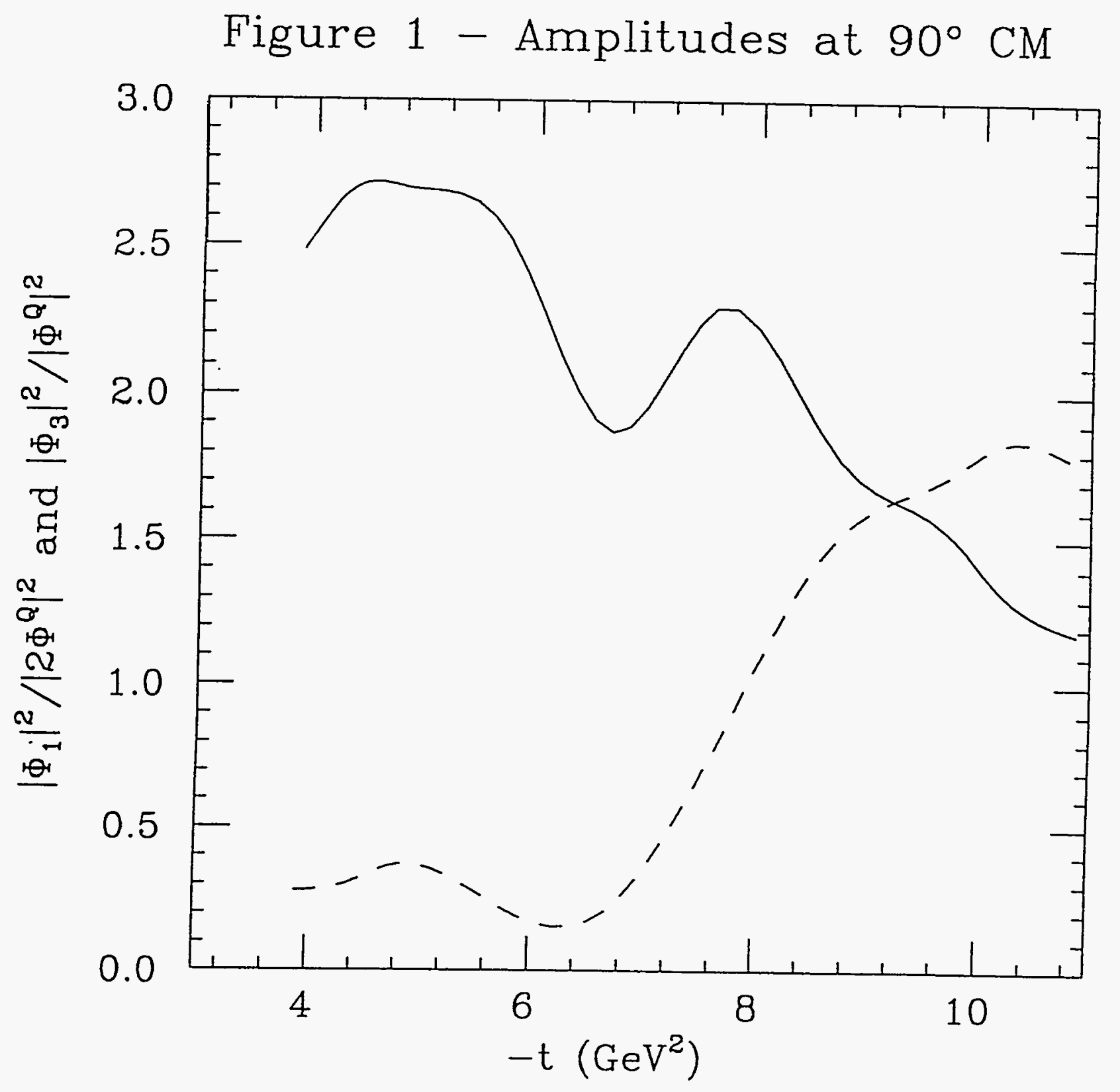

Behavior of the ratios $R_{1} \equiv \frac{\left|\Phi_{1}\right|^{2}}{|2 \Phi Q|^{2}}$ (solid curve) and $R_{3} \equiv \frac{\left|\Phi_{3}\right|^{2}}{|\Phi Q|^{2}}$ (dashed curve) with $-t$, extracted from 90 degree c.m. data.

\section{DISCLAIMER}

This report was prepared as an account of work sponsored by an agency of the United States Government. Neither the United States Government nor any agency thereof, nor any of their employees, makes any warranty, express or implied, or assumes any legal liability or responsibility for the accuracy, completeness, or usefulness of any information, apparatus, product, or process disclosed, or represents that its use would not infringe privately owned rights. Reference herein to any specific commercial product, process, or service by trade name, trademark, manufacturer, or otherwise does not necessarily constitute or imply its endorsement, recommendation, or favoring by the United States Government or any agency thereof. The views and opinions of authors expressed herein do not necessarily state or reflect those of the United States Government or any agency thereof. 